\title{
“LO QUE EL AGUA NOS DEJÓ": INVESTIGACIONES BIOARQUEOLÓGICAS EN LA COSTA SUR DE LA LAGUNA MAR CHIQUITA (CÓRDOBA, ARGENTINA)
}

\section{"BROUGHT WITH THE WATER": BIOARCHAEOLOGICAL RESEARCH ON THE SOUTH COAST OF LAGUNA MAR CHIQUITA (CÓRDOBA, ARGENTINA)}

\author{
Mariana Fabra ${ }^{1}$, Soledad Salega ${ }^{2}$, Claudina V. González² y Aldana Tavarone ${ }^{3}$
}

\section{RESUMEN}

En el presente trabajo se exponen los resultados de los trabajos bioarqueológicos realizados entre 2006 y 2012 en 8 sitios arqueológicos ubicados en la costa sur de la Laguna Mar Chiquita, noreste de Córdoba, Argentina. La intervención en dichos sitios se produjo en el marco de un programa de arqueología pública, solicitada por museos públicos y vecinos de la región. En total, se identificaron restos óseos de 11 individuos adultos. Se presenta la descripción arqueológica de los sitios, la caracterización bioarqueológica y las paleopatologías identificadas en los restos, así como las prácticas mortuorias. En cuanto a la cronología de los hallazgos, se realizaron 6 fechados radiocarbónicos sobre el material esqueletal, que permiten ubicar a los restos entre $4058 \pm 89$ y $487 \pm 45$ años ${ }^{14} \mathrm{CAP}$.

Palabras clave: Arqueología Pública, Bioarqueología, Laguna Mar Chiquita, Córdoba, Argentina

\section{ABSTRACT}

In this paper we describe the results of bioarchaeological research conducted between 2006 and 2012 in 8 archaeological sites located on the southern coast of the Laguna Mar Chiquita, northeast Córdoba, Argentina. Intervention in such sites occurred in the context of archaeological rescue activities requested by public museums and residents of the region. We identified human remains of eleven adult individuals. In this paper we present the description of the archaeological sites, bioarchaeological analysis of the human remains, paleopathology and mortuary practices. We obtained six radiocarbon dates on skeletal material, which allows to locate the remains between $4058 \pm 89$ and $487 \pm 45 \mathrm{C}^{14}$ years BP.

Keywords: Public Archaeology, Bioarchaeology, Laguna Mar Chiquita, Córdoba, Argentina

Tipología: Artículo de Investigación Científica y Tecnológica

Fecha de recepción: 05/03/2014

Fecha de aceptación: 06/08/2014

Forma de citar el artículo: Fabra, M., Salega, S., González, C.V., \& Tavarone, A. (2014). Lo que el agua nos dejó": investigaciones bioarqueológicas en la Costa Sur de la Laguna Mar Chiquita (Córdoba, Argentina). Jangwa Pana, 13, 51 - 64

1. Doctora en Historia, IDACOR/CONICET-Museo de Antropología (Facultad de Filosofía y Humanidades, Universidad Nacional de Córdoba) Argentina. Correo: marianafabra@gmail.com

2. Licenciadas en Historia, Instituto de Antropología de Córdoba-CONICET/Museo de Antropología, Facultad de Filosofía y Humanidades, Universidad Nacional de Córdoba, Argentina. Correo: soledadsalega@gmail.com; cvictoriagonzalez22@hotmail.com

3. Estudiante de ciencias Biológicas. Museo de Antropología (Facultad de Filosofía y Humanidades, Universidad Nacional de Córdoba), Argentina. Correo: aldyt@hotmail.com 


\section{INTRODUCCIÓN}

E n los últimos años se han realizado numerosos hallazgos de materiales arqueológicos -cerámica, materiales líticos, pero particularmente restos óseos humanos en contextos de inhumación- puestos al descubierto por el continuo avance y retroceso de las aguas de la Laguna Mar Chiquita, ubicada en el noreste de la provincia de Córdoba, en el centro de Argentina. Las fluctuaciones en el nivel han sido constantes a lo largo del tiempo; sin embargo, en la década de 1970 el nivel se incrementó en forma drástica debido al aumento de las precipitaciones en la cuenca de captación de los ríos afluentes y la dinámica de los aportes de aguas subterráneas. Estos incrementos y descensos en los niveles de la laguna, y las consiguientes modificaciones de la línea de costa han generado un proceso de erosión continua que ha repercutido en la conservación de los sitios arqueológicos. Esta situación ha requerido que en los últimos años el Programa de Arqueología Pública -PAP- (SEU, Museo de Antropología, FFyH, UNC) realizara 10 rescates arqueológicos para la recuperación sistemática de restos óseos humanos en sitios actualmente costeros, puestos al descubierto por la acción erosiva de las aguas (Fabra et al., 2008, 2013a; Zabala y Fabra, 2012). Los pedidos de intervención fueron realizados por vecinos y museos públicos de la región, con quienes se viene trabajando activamente en el transcurso de los últimos 6 años: el Museo de la región de Ansenuza "Aníbal Montes" de la localidad de Miramar y el Museo Histórico Municipal de La Para, de la localidad homónima. Sobre estas colecciones bioarqueológicas se han iniciado novedosas investigaciones, tendientes a indagar sobre problemáticas vinculadas a la variabilidad genética (Nores et al. 2011), morfológica y epigenética craneofacial (Fabra 2009; 2013b; 2014), cambios y continuidades en la dieta y la salud (Fabra \& Gonzalez 2012), así como en los niveles de actividad física (Salega \& Fabra 2013), prácticas mortuorias (Fabra et al. 2009) y vínculos con otras poblaciones, en función de la evi- dencia arqueomalacológica (Fabra et al. 2012), entre otras. Más recientemente, en un estudio exhaustivo que incluyó el análisis bioarqueológico y genético de 50 individuos de esta región (Fabra et al. 2014), se observó una elevada presencia de patologías dento-alveolares, que sugerirían el consumo de alimentos ricos en hidratos de carbono y azúcares, así como una disminución en los niveles de salud hacia finales del Holoceno tardío y una mayor demanda física por cambios en las actividades de subsistencia o por un proceso de intensificación en la explotación de recursos. También se evidencian continuidades a lo largo del Holoceno en la frecuencia de rasgos epigenéticos y haplogrupos mitocondriales analizados, sugiriendo relaciones biológicas con grupos de la región pampeana, noreste argentino y Patagonia.

En el presente trabajo, y en consonancia con las investigaciones bioarqueológicas llevadas adelante en esta región, interesa presentar puntualmente los trabajos de rescate arqueológico realizados en 8 sitios actualmente costeros, ubicados en la margen sur de la laguna Mar Chiquita, en el noroeste de la región pampeana (provincia de Córdoba, Argentina), así como el análisis bioantropológico de cada uno de los individuos recuperados, desde una perspectiva osteobiográfica (Saul, 1972)

\section{MATERIALES Y MÉTODOS}

Los trabajos se enmarcaron en la arqueología de rescate, por cuanto resultaron en la prospección y excavación de sitios definidos principalmente por la aparición de restos óseos humanos, que se encontraban en riesgo, y que fueron reconocidos $\mathrm{y}$ valorados por parte de vecinos e instituciones culturales de la región. En todos los casos, previo a la excavación, se realizó una prospección con el objetivo de delimitar los sitios, y definir la superficie donde sería necesario excavar. Sólo se excavó en aquellos sectores donde aparecían restos óseos humanos semienterrados, y en peligro de destrucción. La técnica de excavación fue por 
decapage, exponiendo totalmente cada uno de los restos óseos, para luego proceder a su registro y exhumación. En la figura 1 se presenta la ubicación en el mapa de los 8 sitios trabajados en la región.

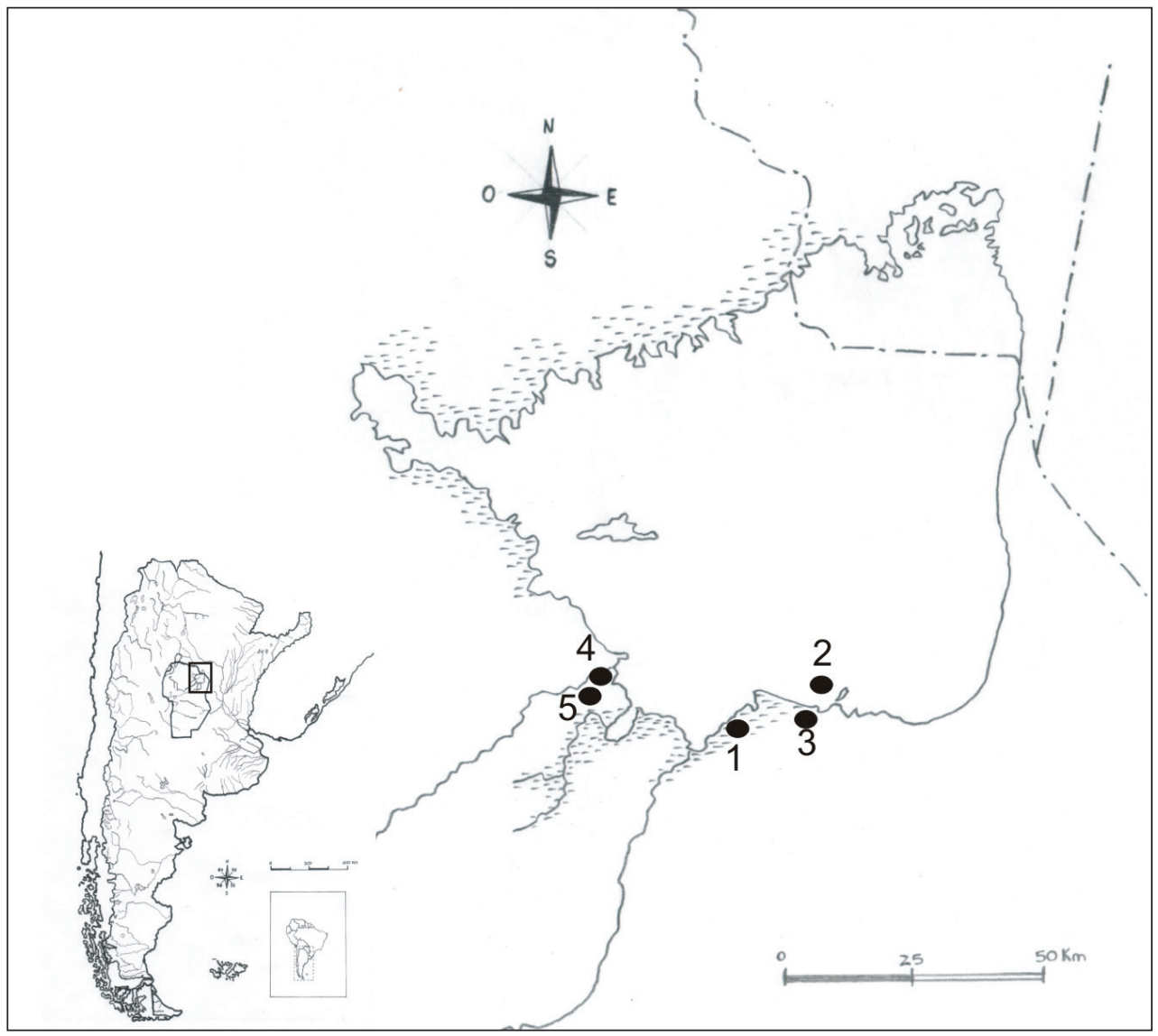

Figura 1. Procedencia de los individuos analizados en el presente trabajo. En un recuadro se señala la ubicación geográfica de la laguna Mar Chiquita, al noreste de la provincia de Córdoba (Argentina). El número entre paréntesis indica la ubicación en el mapa. Sitios: (1) Colonia Müller 1 y 2 (2 individuos), (2) Isla Orihuela 07, 11 y 12 (3 individuos), (3) Orihuela 06 (2 individuos), (4) Estancia La Elisa 06 y 12 (2 individuos), (5) Campo Bocassi-Agua Mansa (2 individuos).

Para el análisis bioantropológico de los restos, se tuvieron en cuenta los procedimientos establecidos por Buikstra y Ubelaker (1994) para la determinación del sexo y estimación de edad de cada uno de los individuos analizados. Se relevaron cuatro bioindicadores dentales en función de su manifestación de origen infeccioso (caries, abscesos y pérdidas dentales antemortem) y de origen metabólico (hipoplasia del esmalte dental) (Lukacs 1989; Buikstra \& Ubelaker 1994; Hillson 2001). Asimismo, se registraron macroscópicamente tres marcadores óseos relacionados con el desarrollo de actividades físicas: nódulos de Schmorl en la columna vertebral (ambas caras de las vértebras), eburnación en las superficies articulares del esqueleto apendicular de 
hombro, codo, muñeca, cadera, rodilla, tobillo y primera articulación metatarso-falángea, y osteofitosis en los sectores anatómicos mencionados (Salega \& Fabra 2013).

\section{RESULTADOS}

\section{Sitio Colonia Müller (CM06)}

Se trata de un sitio al aire libre, distante a $50 \mathrm{~m}$ de la costa de la Laguna Mar Chiquita y ubicado a $8 \mathrm{~km}$. de la ciudad de Miramar, Departamento San Justo, sobre un campo privado del cual se tomó el nombre. Las referencias del sitio fueron dadas por personal del Museo de la Región de Ansenuza "Anibal Montes". Las tareas de rescate se realizaron en octubre de 2006, a partir de la aparición de restos óseos humanos en superficie. En un radio de $20 \mathrm{~m}$ se encontraron restos óseos humanos pertenecientes a un mínimo de dos individuos. El primer conjunto de restos, denominado "Colonia Müller 1" apareció a nivel superficial, parcialmente removido. De acuerdo con las características de la inhumación, se trata de un enterratorio primario simple, en posición decúbito dorsal, con los miembros inferiores hiperflexionados hacia la izquierda, el brazo derecho extendido y el izquierdo semiflexionado sobre la cintura pélvica. La orientación del cuerpo es Este (cráneo)-Oeste (pies). El cráneo se encontraba desplazado de su posición anatómica original. No se encontró material arqueológico asociado que permita inferir contextos culturales y cronología relativa. Para proceder a la determinación del sexo de este individuo se tuvo en cuenta la apófisis mastoidea, el margen supraorbital, la glabela y la eminencia mental, en cuanto a la pelvis se consideraron la escotadura ciática y el surco preauricular. En el caso del cráneo la morfología de estos elementos, permitieron estimar el sexo como femenino. La mayor angulación de la escotadura ciática y del surco preauricular permite también determinar al individuo 1 como femenino. Para la estimación de la edad, no pudo utilizarse la sínfisis púbica, por estar en mal estado de conservación. La evaluación conjunta de la superficie auricular y la superficie esternal de las costillas determinaría una edad promedio de $26 \pm$ 7 años. En cuanto al relevamiento de patologías dento-alveolares, se relevaron 3 piezas dentales y 8 alveolos. Se registraron 2 caries, en primer molar y primer premolar superiores derechos y 1 pérdida dental antemortem. Se observó un desgate muy avanzado en todas las piezas dentales. En cuanto al relevamiento de indicadores de niveles de actividad física, se observó osteofitosis en columna vertebral, principalmente en C3, T5, T11, T12, L1, (grado moderado), y L2 a L5 (grave), y porosidad en T11 y T12 (moderado), así como en L1 a L4 (grave). Los restos del individuo 1 fueron fechados en $1585 \pm 15$ años ${ }^{14} \mathrm{C}$ AP (UCI 39102 , fragmentos postcraneales).

Siguiendo la costa de la laguna hacia el Este, a aproximadamente $300 \mathrm{~m}$ del sitio Colonia Müller 1 se identificó otro conjunto de restos óseos en superficie, al que se denominó "Colonia Müller 2 ". Como en el caso anterior, los restos se encontraron a nivel superficial, a una distancia de 100 $\mathrm{m}$ de la costa actual de la laguna. Del conjunto, se identificó un cráneo de un individuo adulto, sin conexión anatómica con una tibia, peroné y pie articulados. Las partes correspondientes a vértebras, costillas, miembros superiores y cintura pélvica se encontraban ausentes, desintegradas por la exposición al sol y agentes erosivos hídricos y eólicos. Debido al estado de conservación y a la falta de piezas diagnósticas fue difícil determinar el sexo del individuo 2. Sin embargo, se tuvieron en cuenta el ángulo de la mandíbula, el proceso mastoideo izquierdo y la cresta nucal para la determinación del sexo. Considerando estos elementos en conjuntos se determinó el sexo como femenino. En cuanto a la edad, se utilizó el método de obliteración de las suturas craneales (Meindl \& Lovejoy, 1985), el cual permitió estimar un rango de entre 35 y 50 años. No se relevaron patologías en este individuo. 


\section{Sitio Orihuela -Orih06- $\left(30^{\circ} 52 ` 913^{`}\right.$ LS, $062^{\circ} 31^{` 110 `(L)}$}

Los restos óseos humanos fueron recuperados en el mes de julio de 2006, a partir del pedido realizado por el Museo de la Región de Ansenuza "Aníbal Montes". Los restos fueron encontrados a 100 m del margen sur de la Laguna Mar Chiquita, en campos propiedad de la familia Bianco, en el Departamento San Justo. El sitio fue denominado Orihuela (Orih06). El hallazgo lo realizó el Sr. Alberto Rumachella, quien exhumó los restos casi en su totalidad con personal del Museo previo a la realización del rescate. Se recuperó un conjunto de restos óseos humanos conformado por un cráneo y esqueleto postcraneal. El inventario y la determinación del número mínimo de individuos presentes en el conjunto determinó la presencia de restos óseos correspondientes a un individuo adulto, de sexo posiblemente femenino, de entre 20 y 40 años de edad. De acuerdo con el registro de la exhumación, se puede decir que se trata de un enterratorio primario simple individual, en posición flexionada decúbito lateral izquierdo, ubicado el cuerpo de Norte a Sur, con el cráneo orientado hacia el Este. No se recuperó material arqueológico de la sepultura. Sin embargo, cabe destacar que los terrenos donde se produjo el hallazgo se encontraban bajo las aguas de la Laguna hasta el año 2005, situación que provocó la desaparición de niveles superiores de sedimento y los posibles restos arqueológicos asociados a la sepultura. En este individuo se relevaron 8 piezas dentales y 16 alveolos. Se observó una caries de gran tamaño que afectó la corona, cuello y raíz del tercer molar superior derecho y 4 pérdidas dentales antemortem. Se observó un desgaste dental moderado en toda la arcada bucal. También se notó que las epífisis distales de ambos húmeros se encuentran afectadas por eburnación, en grado leve. Se cuenta con un fechado radiocarbónico que ubica a este individuo en $1045 \pm 15$ años ${ }^{14} \mathrm{C}$ AP (UCI 39101, primer metatarso izquierdo).

\section{Sitio Isla Orihuela -IOrih 07- (3051`449`'LS, 62 $27^{\circ} 771^{`}$ LO)}

A partir del pedido realizado por el Museo de la Región de Ansenuza "Aníbal Montes", informando que unos particulares reconocieron restos óseos humanos en superficie, junto con restos dispersos de cerámica arqueológica, loza, vidrio y metal, se realizaron las tareas de rescate. Los restos fueron recuperados en abril de 2007, en un sitio al aire libre ubicado en la costa Este de la Isla Orihuela, Departamento San Justo. Durante el reconocimiento superficial, los restos óseos aparecieron fragmentados, dispersos y parcialmente cubiertos por barro. Debido a las condiciones del sitio, no se trazaron cuadrículas, sino que se recogieron los fragmentos en superficie y se comenzó con la excavación en dos áreas donde se observaba la presencia de cráneos. La excavación de los restos denominados como Individuo 1, sólo resultó en la recuperación de un frontal y fragmentos de parietales.

Las tareas de excavación sobre los restos del Individuo 2, resultaron en la presencia de un esqueleto completo. Este individuo se encontraba inhumado en una sepultura primaria, en decúbito dorsal, con el brazo derecho extendido, el brazo izquierdo semiflexionado con la mano sobre la pelvis, y los miembros inferiores extendidos. Estaba orientado Sur (cráneo) - Norte (pies). No apareció material arqueológico que pudiera ser considerado como ajuar. Aparte de los restos encontrados a nivel superficial, durante la excavación no se recuperaron materiales arqueológicos asociados al enterratorio.

El inventario y la determinación del número mínimo de individuos presentes en el conjunto pusieron de manifiesto la presencia de restos óseos correspondientes a un individuo adulto, de sexo indeterminado (individuo 1), y a un adulto, de sexo masculino, de $47 \pm 12$ años de edad (individuo 2). En el individuo 2 se relevaron 28 piezas 
dentales y 32 alvéolos. Se registraron 3 caries, todas registradas en molares del maxilar superior. Se registró cálculo dental en cantidad moderada en todas las piezas dentales. En la columna vertebral, presenta osteofitosis en C3, C4 (grado leve), T5 a T12 (moderado) y L1 a L4 (grave), así como porosidad en T10, L5 (leve), L3, L4 (moderado), y T11 a L2 (grave). Asimismo, se registró la presencia de nódulos de Schmorl en T11, T12, L1, L3 y L4. Se realizó un fechado radiocarbónico sobre el individuo 2, que lo ubica en $761 \pm 41$ años $\mathrm{C}^{14} \mathrm{AP}$ (AA93746, metacarpo y falange media derechos).

\section{Estancia La Elisa 09 -EE09- $\left(30^{\circ}\right.$ $43 ` 558$ `LS, $62^{\circ} 59^{`} 752$ ` $\left.\mathrm{LO}\right)$}

Los restos fueron descubiertos en mayo de 2009 por el señor Carlos Pamater, vecino de la localidad de La Para, Departamento Río Primero, a 150 metros de la costa actual de la laguna Mar Chiquita, en un paraje denominado Estancia La Elisa, distante aproximadamente $20 \mathrm{~km}$. de dicha localidad. El particular dio aviso de la aparición de los restos al personal policial de la localidad y al Museo Histórico Municipal de La Para, quienes convocaron a su vez al PAP, que realizó los trabajos de exhumación el día 31 de mayo. El cráneo y una mandíbula fueron removidos por personal del Museo de La Para y la policía de la misma localidad (Departamental Santa Rosa Río Primero).

Es un sitio al aire libre, definido a partir de la aparición de restos óseos en superficie. Los restos aparecieron a unos $20 \mathrm{~cm}$ del suelo actual, $\mathrm{y}$ se encontraron sobre un sedimento limo arcilloso. Se trata de una inhumación primaria simple, en decúbito dorsal, con los miembros superiores flexionados sobre el torso, y los miembros inferiores flexionados hacia la izquierda. Asociado a este individuo, se encontró un fragmento lítico de cuarzo que corresponde a un ápice de una punta de proyectil fragmentada, ubicado entre el omóplato y las costillas izquierdas. Si bien no se han observado lesiones perimortem en estas partes anatómicas no se descarta un contexto de violencia interpersonal para este caso, como ha sido discutido previamente (Fabra et al. 2013c). Los análisis bioantropológicos determinaron que se trata de un individuo de sexo masculino, de entre 42 y 46 años de edad al momento de su muerte. Este individuo presenta 4 piezas dentales y 32 alveolos. No presenta caries, abscesos e hipoplasias del esmalte dental, en cambio presenta gran cantidad de pérdidas dentales antemortem, un total de 14, tanto de incisivos como de molares, superiores e inferiores. También presenta osteofitosis (grado leve) en T10 a T12, así como porosidad en T11, T12, y L1 a L5 (leve). Se realizó un fechado radiocarbónico sobre el individuo 1 , que lo ubica en $1890 \pm 49$ años ${ }^{14} \mathrm{C}$ AP (AA102656, falange proximal).

\section{Isla Orihuela Playa Sudeste -IOrih11 PS}

Es un sitio al aire libre ubicado en la Isla Orihuela, en la laguna Mar Chiquita, departamento San Justo. En superficie se observa una dispersión de fragmentos de cerámica arqueológica. La excavación de este sitio se realizó en abril de 2011, definiéndose una grilla de $12 \mathrm{~m}$ por $12 \mathrm{~m}$ a partir de la cual se establecieron un total de 36 cuadrículas de $2 \mathrm{~m}$ por $2 \mathrm{~m}$ cada una. Se excavaron tres cuadrículas siguiendo la metodología de la matriz de Harris para la definición de unidades estratigráficas. Se recuperó abundante material óseo faunístico, malacológico y cerámico sin decoración.

Hacia el norte del grillado, se encontraron restos óseos humanos (cara y cráneo fragmentados), por lo que se trazó una cuadrícula de $1 \mathrm{~m}$ por $2 \mathrm{~m}$ siguiendo la posible articulación anatómica del esqueleto. El individuo se encontraba en un entierro primario simple, en decúbito dorsal, extendido, con el cráneo hacia el sur y pies al oeste, mientras que las órbitas estaban orientadas al 
oeste. Los miembros superiores están extendidos al costado del cuerpo; no se encontraron las manos, pero por la posición de cúbitos y radios el brazo y mano derechos habrían pasado por sobre la pelvis, y el brazo y mano izquierda por debajo. En el caso de los miembros inferiores se encuentran extendidos, con la rodilla izquierda ligeramente flexionada hacia el plano lateral. Los restos se encuentran en un estado de conservación regular a malo. Por debajo de la epífisis proximal del húmero izquierdo apareció una uña de Rhea (posiblemente juvenil). Asimismo, se registraron fragmentos de cerámica.

Los análisis bioantropológicos permitieron determinar que se trata de un individuo de sexo femenino, adulto, sin poder acotar un rango de edad debido al mal estado de conservación de los elementos óseos diagnósticos. Este individuo presenta sólo una pieza dental (canino superior izquierdo) y 16 alveolos. Presenta gran cantidad de pérdidas dentales antemortem, un total de 11 , y no presenta abscesos.La columna vertebral se encuentra afectada por osteofitosis en C4, C5, T7, T8 y T9 (grado leve) y en T11, T12 y L1 (moderado), y por porosidad (grado leve) en T11, T12, y L1 a L5. Se realizó un fechado radiocarbónico sobre el individuo 1, que lo ubica en $487 \pm 45$ años $\mathrm{C}^{14} \mathrm{AP}$ (AA102657, dos carpos izquierdos).
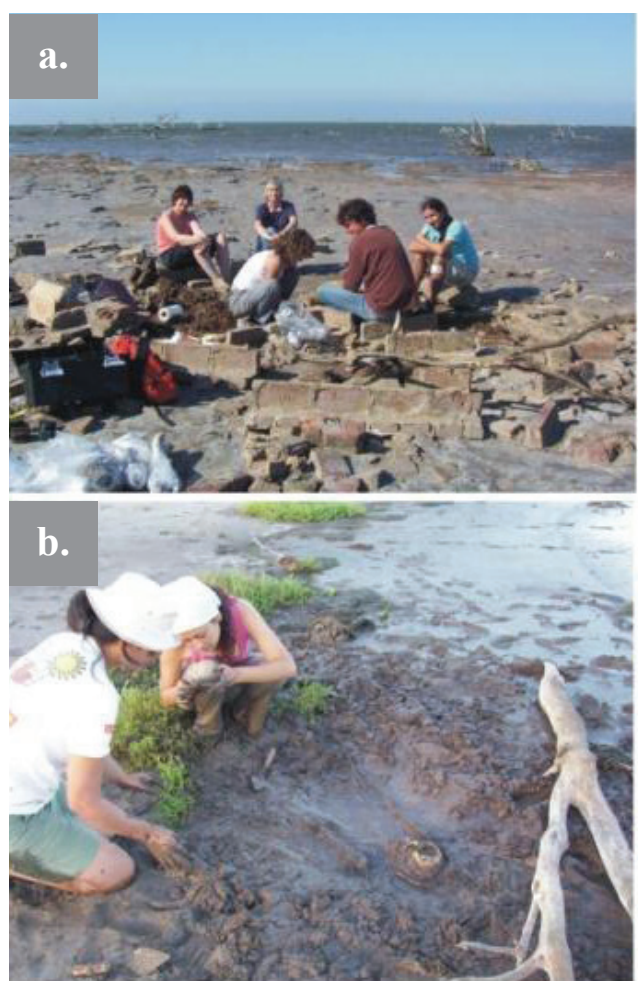

Figura 2a) Sitio Colonia Müller, 2b) Sitio Isla Orihuela, 2c) Sitio Orihuela, 2d) Sitio Estancia La Elisa 09.

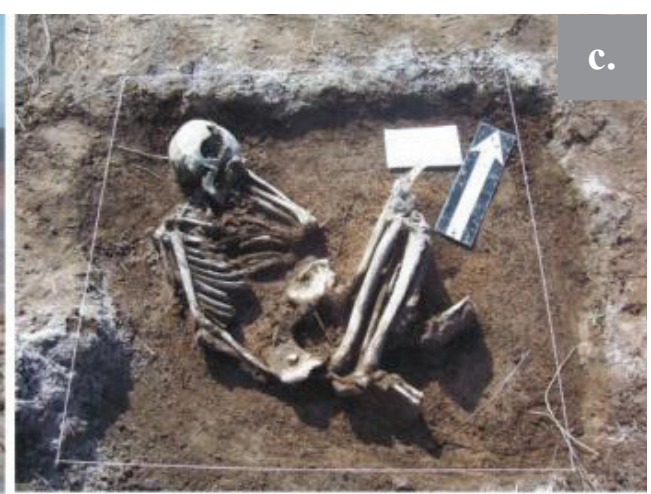

d.

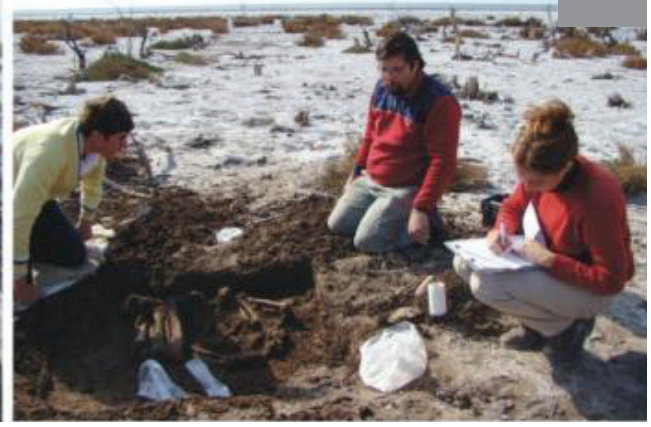


"LO QUE EL AGUA NOS DEJÓ”: INVESTIGACIONES BIOARQUEOLÓGICAS EN LA COSTA SUR DE LA

\section{Campo Bocassi-Agua Mansa -CB-AM 12- $\left(30^{\circ} 42^{`} 400^{`} \mathrm{LS}, 63^{\circ} 01^{`} 490^{`} \mathrm{LO}\right)$}

El Museo Histórico Municipal de La Para convocó al PAP debido a la aparición de restos óseos en cercanías de la costa de la laguna Mar Chiquita, en el campo conocido como Agua Mansa, propiedad de la familia Bocassi. Las tareas de rescate arqueológico se llevaron a cabo en el mes de mayo de 2012. En superficie se observaba parte de la bóveda craneal, meteorizada por su exposición al sol y el agua, a partir de la cual se trazó una cuadrícula siguiendo la posible ubicación del resto del esqueleto y se procedió a su excavación.

Se trata de una inhumación primaria simple, con el individuo en posición decúbito dorsal, con los miembros flexionados hacia el lado izquierdo. El cráneo está orientado hacia el sudoeste y los pies hacia el noreste, con las órbitas hacia el oeste. Se encuentra parcialmente desarticulado en los pies, con algunos elementos óseos dispersos. El estado de conservación de los restos es regular y se encuentra afectado por la acción de raíces en el sector de costillas y pelvis. No se encontraron elementos que puedan considerarse como ajuar. Los análisis bioantropológicos determinaron que se trata de un individuo de sexo masculino, adulto, sin poder acotar un rango de edad debido al mal estado de conservación de los elementos óseos diagnósticos. Este individuo tiene 19 piezas dentales presentes y 31 alveolos. Presenta un total de 6 caries ubicadas en molares y premolares y se registraron un total de 7 abscesos apicales. Se registró un avanzado desgaste dental en todos los dientes presentes. El individuo presenta osteofitosis (grado leve) en las epífisis proximales de cúbito izquierdo y derecho, en ambas epífisis del radio izquierdo, y en semilunares y escafoides. Además, presenta porosidad (moderada) en las epífisis distales de los húmeros. No se cuenta aún con fechado radiocarbónico para este individuo, pero se estima que correspondería al Holoceno tardío (2500-300 años AP).

\section{Isla Orihuela Costa Sur -IOrih CS12- $\left(30^{\circ}\right.$ $\left.51^{`} 523^{`} \mathrm{LS}, 062^{\circ} 27^{`} 811^{`} \mathrm{LO}\right)$}

Se trata de un sitio al aire libre, excavado en el año 2012, ubicado en la costa sur de la laguna Mar Chiquita, en la isla denominada Orihuela, cercana a localidad de Miramar, Departamento San Justo. Se trata de un único individuo incompleto, se constató la ausencia de huesos largos de miembros superiores e inferiores y ausencia de cráneo. Se encontraba articulado en pelvis, sector inferior de columna vertebral y pies. Debido al estado de conservación no se pudo determinar sexo ni edad. Dado el estado de conservación de los restos esqueletales, no pudieron relevarse paleopatologías. Aún no contamos con información radiocarbónica, pero suponemos corresponde al Holoceno tardío final (1500-500 años AP) en función de las características tecnológicas del material cerámico hallado en superficie.

\section{Estancia La Elisa -EE 012- $\left(30^{\circ}\right.$ 44 `361` 'LS, $062^{\circ} 56$ `628` LO)}

Se trata de un sitio al aire libre, excavado en 2012, ubicado en la costa Oeste de la Laguna Mar Chiquita, a unos $80 \mathrm{~m}$ de la actual costa, en un campo privado denominado Estancia La Elisa. En superficie se recuperó gran cantidad de material cerámico y lítico. Se trata de restos óseos humanos desarticulados y dispersos posiblemente por acción del agua, pertenecientes a un único individuo, denominado 1, inhumado en una fosa primaria en posición decúbito dorsal, se observó articulación de la columna vertebral, pelvis y sacro, húmeros y omóplatos derechos e izquierdos, fémur, fragmento de tibia y pie izquierdos también articulados. Se encontraron radios, cubitos y peronés desarticulados, dispersos en un radio de $2 \mathrm{~m}$. La mandíbula se encontró fragmentada y por encima del sacro. A unos $10 \mathrm{~m}$ de distancia en dirección norte se encontró un cráneo que posiblemente corresponda a este individuo. Según 
el análisis bioantropológico se pudo determinar que se trata de un individuo masculino, de entre 16 y 20 años de edad. Se relevaron un total de 23 piezas dentales y 32 alveolos. No se registraron caries, abscesos ni pérdidas dentales antemortem. Sin embargo, se observaron líneas de hipoplasias en caninos. Aún no se realizó el relevamiento de marcadores de estrés funcional.

A $200 \mathrm{~m}$ en dirección Norte del individuo 1, se identificaron restos correspondientes a otro individuo, denominado $2\left(30^{\circ} 44^{\prime} 180^{\prime \prime}\right.$ LS, $062^{\circ} 56^{\prime} 813^{\prime}$ LO). Se trata de un único individuo completo, en buen estado de conservación, inhumado en una fosa primaria, orientado en dirección Oeste-Este, posición sedente, con los miembros inferiores hiperflexionadas sobre el torso, el miembro superior derecho semiflexionado debajo del fémur derecho y el miembro superior izquierdo semiflexionado por debajo de fémur, tibia y peroné izquierdos. Según el análisis bioantropológico se pudo determinar que se trata de un individuo masculino, adulto, de entre 35 y 46 años de edad. Hasta el momento solamente se ha realizado el relevamiento de patologías dentales en este individuo. Presenta un total de 13 piezas dentales y 28 alveolos. Se registraron una caries en superior derecho y un absceso periapical en primer molar inferior izquierdo. Se observó un elevado desgaste dental en todas las piezas dentales. Se realizó un fechado radiocarbónico sobre este individuo, que lo ubica en $4058 \pm 89$ años ${ }^{14} \mathrm{C}$ AP (AA102655, falange proximal derecha).
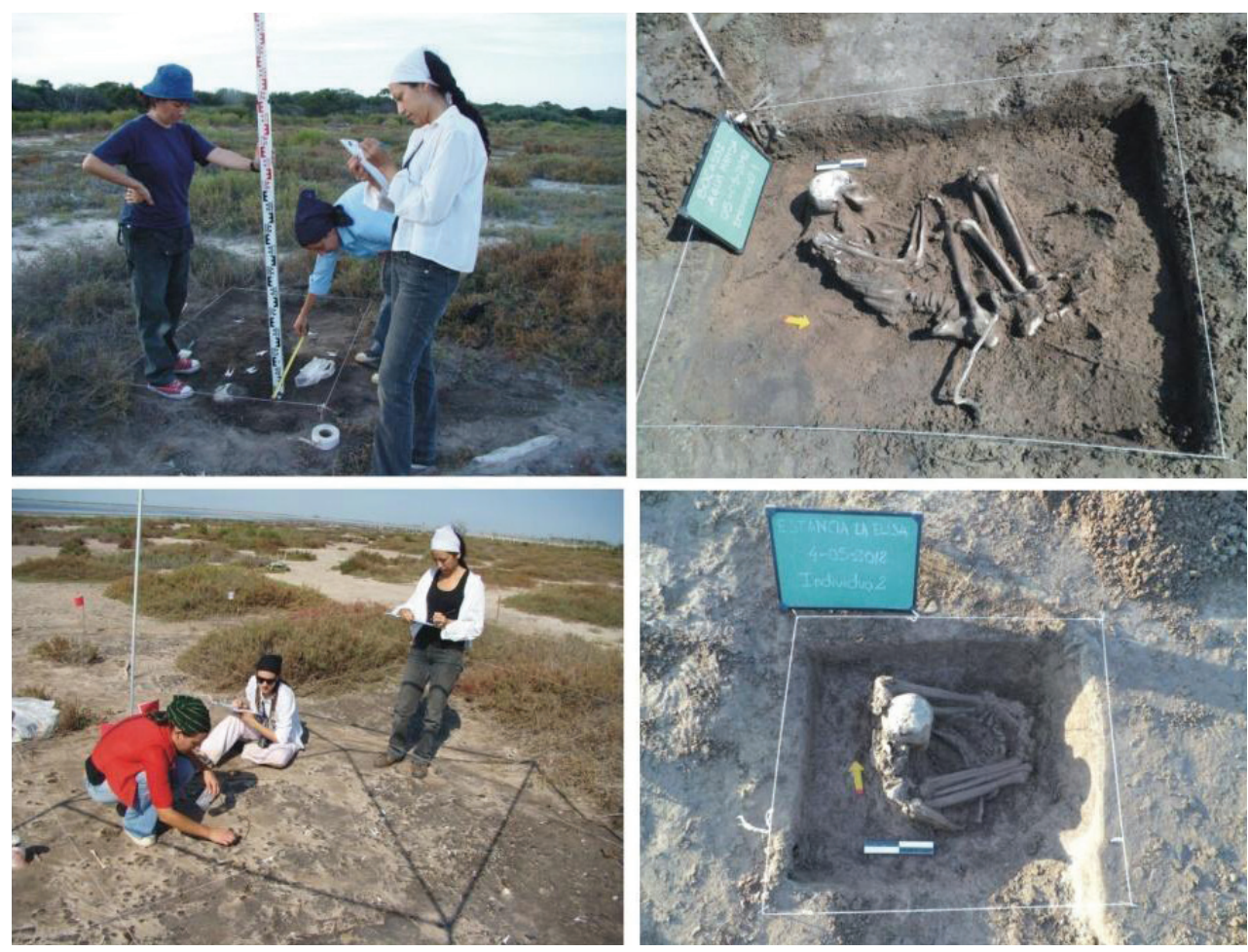

Figura 3a y b). Sitio Isla Orihuela 11 Playa Sudeste, 3c). Sitio Campo Bocassi-Agua Mansa 12, 3d). Sitio Estancia La Elisa 012, individuo 2. 
A modo de síntesis, en la tabla 1 se sintetiza la información bioantropológica y cronológica de los 9 individuos recuperados.

Tabla 1. Información bioantropológica y cronológica de los 11 individuos recuperados.

\begin{tabular}{|c|c|c|c|c|c|c|c|c|}
\hline Sitio & Individuo & Sexo & Edad & C14 (AP) & ID lab. & $\begin{array}{l}d^{13} C_{\text {PDB }} \\
\text { (\%) }\end{array}$ & $\mathbf{d}^{15} \mathrm{~N}_{\text {air }}(\%)$ & A destacar \\
\hline $\begin{array}{c}\text { Colonia } \\
\text { Muller } 06 \\
\text { sitio } 1 \\
\end{array}$ & CM06 I1 & $\mathrm{F}$ & $26 \pm 7$ & $1585 \pm 15$ & UCl 39102 & $-13,7$ & 9,6 & \\
\hline $\begin{array}{l}\text { Colonia } \\
\text { Muller } 06 \\
\text { sitio } 2\end{array}$ & CM06 I2 & $\mathrm{F}$ & $35-50$ & - & - & - & - & - \\
\hline Orihuela 06 & Orih06 I6 & $\mathrm{F}$ & $20-40$ & $1045 \pm 15$ & UCI 39101 & $-14,0$ & 10,0 & \\
\hline $\begin{array}{l}\text { Isla Orihuela } \\
07\end{array}$ & IOrih07 I2 & M & $47 \pm 12$ & $761 \pm 41$ & AA93746 & $-15,2$ & - & - \\
\hline $\begin{array}{l}\text { Isla Orihuela } \\
07\end{array}$ & IOrih07 I1 & - & A & - & - & - & - & - \\
\hline $\begin{array}{c}\text { Estancia La } \\
\text { Elisa } 09 \text { sitio } \\
\text { El Belga }\end{array}$ & EE09 I1 & M & $42-46$ & $1,890 \pm 49$ & AA102656 & $-16,9$ & - & $\begin{array}{c}\text { Contexto } \\
\text { de violencia } \\
\text { interpersonal } \\
\text { ápice de punta } \\
\text { de proyectil } \\
\text { lítica asociada a } \\
\text { esqueleto } \\
\end{array}$ \\
\hline $\begin{array}{l}\text { Isla Orihuela } \\
11 \text { Playa } \\
\text { Sudeste }\end{array}$ & IOriH11 PS & $\mathrm{F}$ & A & $487 \pm 45$ & AA102657 & $-14,4$ & - & - \\
\hline $\begin{array}{c}\text { Isla Orihuela } \\
\text { Costa Sur } \\
12 \\
\end{array}$ & IOrih12 & - & - & - & - & - & - & - \\
\hline $\begin{array}{c}\text { Estancia La } \\
\text { Elisa } 12\end{array}$ & EE12 I1 & M & $16-20$ & - & - & - & - & - \\
\hline $\begin{array}{c}\text { Estancia La } \\
\text { Elisa } 12\end{array}$ & EE12 I2 & M & A & $4,058 \pm 89$ & AA102657 & $-16,5$ & - & - \\
\hline $\begin{array}{c}\text { Campo } \\
\text { Bocassi- } \\
\text { Agua Mansa } \\
12 \\
\end{array}$ & CB-AM12 I1 & M & A & - & - & - & - & - \\
\hline
\end{tabular}

Referencias: F (femenino), M (masculino), A (adulto); IDLab: UCI (W. M. Keck Carbon Cycle Accelerator Mass Spectrometry Laboratory, University of California, Irvine), AA (NSF-Arizona AMS Laboratory, University of Arizona) 


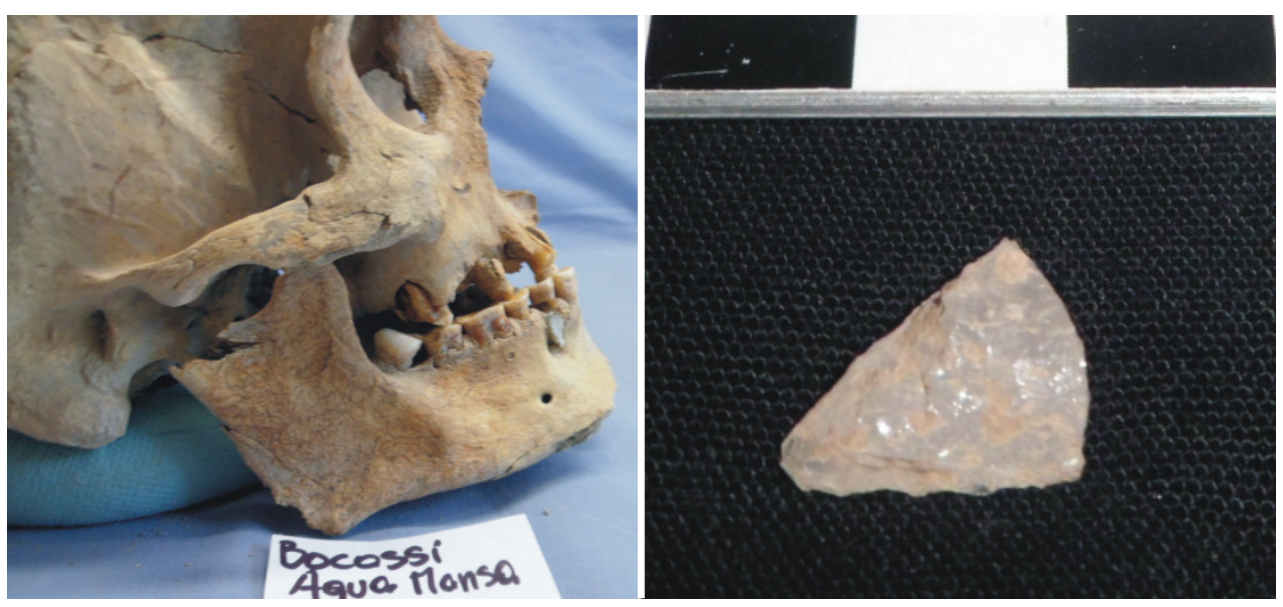

Figura 4. a) Absceso periapical en segundo molar derecho del maxilar. Solo se observa una maloclusion del tercer molar inferior derecho. Sitio Campo Bocassi-Agua Mansa 12, Individuo 1..b) Apice de punta de proyectil de cuarzo asociada a individuo 1, sitio Estancia La Elisa 09.

\section{DISCUSIÓN Y CONCLUSIONES}

Estos trabajos, si bien enmarcados en la arqueología de rescate y acotados en tiempo y espacio, fueron los primeros aportes no sólo para iniciar líneas de investigación novedosas en la región, desde una perspectiva bioarqueológica (Fabra, 2009, 2013a, 2013b; Fabra et al., 2012; Fabra \& González, 2012; Salega \& Fabra, 2013) y arqueomalacológica (Gordillo \& Fabra, 2013; Fabra et al. 2012) en función de las características de los materiales recuperados, sino fundamentalmente en lo que respecta a acciones tendientes a la recuperación y protección del patrimonio arqueológico regional.

Tal como menciona Gómez Otero (2011) para casos similares en la provincia de Chubut (Argentina), el trabajo de rescate arqueológico de restos óseos humanos posibilita su posterior estudio y análisis desde una perspectiva bioarqueológica, brindando información sobre aspectos usualmente no conocidos de las poblaciones originarias. En el caso del noreste de la provincia de Córdoba, nuestras investigaciones pusieron de manifiesto una ocupación continua de la región de al menos 4500 años AP, cambios en cuanto a la composición biológica de las comunidades que la habitaron, sugiriendo relaciones con poblaciones de la región pampeana, Patagonia y el noreste argentino (Nores et al., 2011; Fabra, 2013b, Fabra \& Demarchi, 2013). También hemos podido inferir, a partir del relevamiento de ciertos indicadores óseos y dentales, que en siglos previos a la conquista española se habría producido un desmejoramiento en la calidad de vida, reflejado en la realización de actividades físicas más demandantes, a menor edad (Salega \& Fabra, 2013) y en episodios de estrés metabólico-sistémico (Fabra \& González, 2012). 
En lo que respecta al aporte concreto del análisis de los materiales bioantropológicos recuperados en los sitios presentados en este trabajo, se pueden mencionar los siguientes aspectos: por un lado, los individuos presentan patologías en frecuencia y grado similar a lo detectado en otros casos analizados para la zona, lo cual permite reforzar el patrón observado de baja o moderada prevalencia de caries, hipoplasias del esmalte o pérdidas dentales antemortem, coincidente con una economía de tipo cazadora-recolectora o mixta (Fabra \& Gonzalez, 2012), así como incremento de lesiones osteoartríticas (osteofitosis y nódulos de Schmorl) en individuos jóvenes, en momentos previos a la conquista española (Fabra et al. 2012). Respecto a la presencia de indicadores de estrés metabólico, solamente se relevaron hipoplasias del esmalte en un individuo joven, del cual no se posee cronología absoluta.

Sin embargo, estos casos también presentan particularidades que merecen ser destacadas. Por un lado, la forma de inhumación registrada en el individuo denominado 2 del sitio Estancia La Elisa 012 no solamente es novedosa para la región (posición sendente) sino que además representa el individuo más completo y antiguo recuperado en toda la provincia de Córdoba. Si bien hay fechados realizados sobre restos óseos humanos con fechas previas, también para la zona, de 4500 años AP, por tratarse exclusivamente de calotas no se contaba con información sobre las modalidades de entierro ni habían podido realizarse estudios vinculados con paleopatologías o dieta, como sí lo permite este caso. En la provincia de Córdoba, las modalidades de entierro más antiguas registradas datan de alrededor de 3000 años AP, y corresponden a individuos inhumados en el sitio Agua de Oro, ubicado en el piedemonte de las Sierras de Córdoba, aproximadamente a $200 \mathrm{~km}$. de los sitios analizados en este trabajo (Fabra, 2000; Fabra et al. 2009). Esos individuos presentaban inhumaciones secundarias múltiples. La modalidad de inhumación de tipo sedente con hiperflexionamiento de los miembros, no registrada previamente en entierros tanto en la región serrana como en las llanuras del noreste de la provincia, suma elementos para pensar en dinámicas sociales complejas para las sociedades cazadoras-recolectoras que habitaron el centro de Argentina en el Holoceno medio.

Otro elemento interesante de destacar es el hallazgo de un ápice de proyectil de cuarzo en asociación directa con el individuo 1 del sitio Estancia La Elisa 09, que sugiere un contexto de violencia interpersonal. El registro arqueológico (Laguens y Bonnin 2009) y bioarqueológico (Fabra \& González 2012; Fabra et al. 2012, 2014) regional sugiere que hacia el Holoceno tardio final (1000500 años AP) se habría producido un desmejoramiento en las condiciones y la calidad de vida de las poblaciones que ocuparon los valles, sierras y llanuras de la provincia de Córdoba, así como un crecimiento demográfico y una explotación más intensiva de los espacios, generando una presión sobre los recursos disponibles. Este proceso se habría acentuado hacia el $500 \mathrm{AP}$, derivando en situaciones de circunscripción ambiental y social que habrían conducido al surgimiento de potenciales conflictos sociales por nuevos territorios para el asentamiento y la explotación de recursos (Laguens y Bonnin 2009).Si bien en este trabajo se presenta un único caso con evidencias que hacen pensar en contextos de violencia, el fechado cercano a comienzos de la era cristiana abre interesantes planteos y perspectivas para pensar en las causas que pueden haber generado estas situaciones de conflicto en sociedades de pequeña escala.

El registro y relevamiento de sitios hasta el momento no conocidos, la recuperación de restos óseos humanos y otras materialidades que de otra forma se hubieran perdido o destruido, la información cronológica y el trabajo conjunto con comunidades locales e instituciones culturales de la región no sólo durante los trabajos de campo sino fundamentalmente en talleres, donde se discute y se realiza una puesta en común de los saberes producidos desde la academia y las propias 
concepciones acerca del pasado local (Aichino et al. 2012; Zabala \& Fabra, 2012), son aspectos que merecen ser destacados desde una perspectiva pública de la arqueología. A futuro, se incorporarán estas muestras a los estudios poblacionales que se están realizando para esta región, para discutir aspectos vinculados con cambios y continuidades en la dieta, a partir del análisis de isotopos estables del carbono y del nitrógeno, o relaciones biológicas entre poblaciones, aspectos no abordados en el presente trabajo.

\section{AGRADECIMIENTOS}

Las autoras desean agradecer muy especialmente a los directivos y al personal del Museo Aníbal Montes de la localidad de Miramar, y al Museo Histórico Municipal de La Para, la Dirección de Seguridad Náutica, así como a sus municipios y vecinos, por el constante interés y compromiso en la recuperación, protección y puesta en valor de sitios arqueológicos cercanos a la Laguna Mar Chiquita. Sin su participación no hubiera sido posible la realización de estas tareas. A los compañeros del PAP y a los alumnos de la carrera de Antropología que participaron en los trabajos de campo y análisis de laboratorio. A la doctora Guaciara Dos Santos (W. M. Keck Carbon Cycle Accelerator Mass Spectrometry Laboratory, University of California, Irvine) por la realización de 2 fechados radiocarbónicos mediante convenio de colaboración. Al Dr. Eduardo Piovano, por la realización de 1 fechado en NSF-Arizona AMS Laboratory, University of Arizona, en el marco de un proyecto bajo su dirección. A los evaluadores del trabajo, quienes realizaron valiosas sugerencias que permitieron mejorar la presentación de los datos y los resultados de los análisis. Estos trabajos forman parte del Programa de Arqueología Pública (SEU, Museo de Antropología, FFyH, UNC) y fueron parcialmente financiados por CONICET (PIP 2011-2013, 114 20100100164) y el MINCyT (PID 2010, Res.113).

\section{REFERENCIAS BIBLIOGRÁFICAS}

Aichino, G.L., De Carli M.C., Zabala M.E. \& Fabra M. (2012). Procesos de activación y valoración del patrimonio arqueológico a través de la Cartografía Social. EXT: Revista de Extensión de la Universidad Nacional de Córdoba, 3:1-27. Buikstra, J. \& Ubelaker D. (editores) (1994). Standards for Data Collection from Human Skeletal Remains. Proceedings of a seminar at The Field Museum of Natural History, Arkansas Archaeological Survey Research Series, Nro. 44.

Fabra, M. (2000). Rescatando el patrimonio arqueológico de la provincia de Córdoba: la arqueología de rescate como medio para revalorizar el pasado. Informe final de beca de extensión universitaria (proyecto 16E), Universidad Nacional de Córdoba. Inédito.

Fabra, M. (2009). El poblamiento prehispánico de Córdoba: una interpretación a partir de evidencias bioantropológicas. Universidad Nacional de Córdoba.

Fabra, M. (2013a). Entre el mar y las llanuras: bioarqueología de los primeros pobladores de la región de Ansenuza. En: Nuestros ancestros ribereños: actualización en Antropología y Arqueología de la región de Ansenuza (Córdoba). (pp. 1-15) Museo Histórico Municipal de La Para, Córdoba Fabra, M. (2013b). Variación epigenética craneofacial y dinámica evolutiva de las poblaciones humanas del norte de la región pampeana durante el Holoceno tardío. Revista Cuadernos del Instituto Nacional de Antropología y Pensamiento Latinoamericano, edición especial I Congreso Internacional de Arqueologia de la Cuenca del Plata, 1 (1): 74-86.

Fabra, M., S. Salega, C.V. González \& Robin, S. (2013c). Casos de violencia interpersonal en poblaciones del piedemonte y las llanuras de Cordoba, Argentina. Reunion de la Asociacion de Paleopatologia en Sudamerica -PAMinSA V-, ISBN 2346-0598, Programa de Antropología, Facultad de Humanidades, Universidad del Magdalena, Colombia, Pp.129. 
Fabra, M. (2014). Historia de las poblaciones prehispánicas del sector austral de las Sierras Pampeanas: variabilidad morfológica y modelos arqueológicos. E-Book. Universidad Nacional de Córdoba, Córdoba

Fabra, M., S. Salega \& González C.V. (2009). Comportamiento mortuorio en poblaciones prehispánicas de la región austral de las Sierras Pampeanas durante el Holoceno. Revista Arqueología 15:165-186.

Fabra, M., Salega S., González C.V., Smeding R. \& Pautassi E. (2008). Arqueología de rescate en la costa sur de la Laguna Mar Chiquita: sitio arqueológico El Diquecito. Memorias del Pueblo: Revista del Museo Histórico Municipal La Para. 8 (8):37-46.

Fabra, M., Gordillo S. \& Piovano E.L. (2012). Arqueomalacología en las costas de Ansenuza: análisis de una almeja nacarífera (Anodontites trapesialis) hallada en contexto funerario del sitio El Diquecito (Laguna Mar Chiquita, Córdoba). Revista Arqueología 18: 257-266.

Fabra, M. \& González C.V. (2012). Diet and oral health of populations that inhabited central Argentina (Córdoba province) during late Holocene. International Journal of Osteoarchaeology. DOI: 10.1002/oa.2272

Fabra, M., González C.V. \& Salega S. (2012). Modos de vida e historia biológica de poblaciones de las Sierra y Llanuras de Córdoba (Argentina): aproximaciones desde el registro bioarqueológico. Revista Argentina de Antropología Biológica, 14:87-104.

Fabra, M., \& Demarchi, D. A. (2013). Análisis morfogeométrico aplicado al estudio de los patrones espaciales y temporales de variación morfológica craneofacial en poblaciones del centro de Argentina. Cuadernos de Instituto de Antropología y Pensamiento Latinoamericano, edición especial I Congreso Internacional de Arqueologia de la Cuenca del Plata, 1 (1): 87-101.

Fabra, M., Nores R., Salega S., \& Gonzalez, C. V. (2014). Entre las Sierras y el Mar: investigaciones bioarqueológicas en el noroeste de la región pampeana (costa sur de la laguna Mar Chiquita, Cordoba, Argentina). Avances recientes en la Bioarqueologia Sudamericana, Luna, L. Aranda, C. y J. Suby (eds.). En prensa

Gómez O. J. (2011). La importancia de rescatar los enterratorios humanos en riesgo: experiencias en el nordeste de la provincia de Chubut. Cazadores-recolectores del Cono Sur, Revista de Arqueología, 5: 15-34, 2011.

Gordillo, S. \& Fabra M. (en prensa). El uso de moluscos y caracoles por parte de poblaciones prehispánicas que habitaron el Mar de Ansenuza en el norte cordobés. Revista del Museo Histórico Municipal de La Para.

Hillson S. (2001). Recording dental caries in archaeological human remains. International Journal of Osteoarchaeology 11: 249-289.

Lukacs, J. R. (1989). Dental Paleopathology: Methods for reconstructing dietary patterns. En M. Iscan y K. Kennedy (eds.), Reconstruction of life from the skeleton: 261-286. New York.

Meindl, R. \& Lovejoy, O. (1985) Ectocranial Suture Closure: A Revised Method for the Determination of Skeletal Age at Death Based on the Lateral-Anterior Sutures. American Journal of Physical Anthropology 68:57-66.

Nores, R., Fabra, M., \& Demarchi, D. A. (2011). Variación temporal y espacial en poblaciones prehispánicas de Córdoba. Análisis de ADN antiguo. Revista del Museo de Antropología 4: 187-194.

Saul, F. P. (1972). The Human Skeletal Remains of Altar de Sacrificios: An Osteobiographic Analysis. The Peabody Museum, Cambridge.

Salega, M.S., \& Fabra M. (2013). Niveles de actividad física en poblaciones de las sierras y las llanuras de la provincial de Córdoba (Argentina) durante el Holoceno tardío. Relaciones, 38 (2): 401-420.

Zabala, M. \& Fabra M. (2012). Estrechando vínculos entre "comunidades" en torno al patrimonio arqueológico. Las practicas extensionistas desde un programa de Arqueología Publica. Revista de Arqueología Publica, NEPAM/ UNICAMP, 6: 39-53. 